\title{
Metanálise de pesquisas sobre inundações urbanas: identificação de fatores causais e métodos empregados em estudos recentes
}

\author{
Meta-analysis of urban flooding researches: identification of causal factors and used methods on recent studies

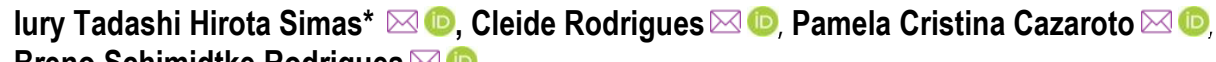 \\ Breno Schimidtke Rodrigues $\bowtie$ (i)
}

Departamento de Geografia, Faculdade de Filosofia, Letras e Ciências Humanas, Universidade de São Paulo, São Paulo, SP, Brasil

Recebido (Received): 15/05/2021

E-mail: cleidrig@usp.br (CR); pamela.cazaroto@gmail.com (PCC);

Aceito (Accepted): 28/06/2021

brenoschmidtke@usp.br (BSR)

*E-mail para correspondência: iurysimas@usp.br

Resumo: No contexto de pesquisa mais ampla, com foco na investigação de fatores causais para ocorrências de inundações nas bacias urbanizadas de São Paulo, foi realizada pesquisa de metanálise sobre estudos que enfocaram inundações urbanas em diferentes perspectivas de análise dos campos do conhecimento correlatos (geomorfologia, climatologia, hidrologia, etc.). Buscou-se identificar relações entre conceitos mais associados, metodologias mais empregadas e fatores causais mais apontados. A metanálise foi realizada em duas etapas: uma mais extensiva, sobre base mundial de periódicos Web of Science, e uma de maior detalhe, sobre bases nacionais de periódicos e repositórios de teses e dissertações. Os dados da primeira etapa foram analisados por meio de mapeamento bibliométrico e os da segunda foram fichados e analisados qualitativamente. A representatividade de pesquisas sobre inundações urbanas com origem em países do meio tropical foi considerada significativamente baixa. Na subárea da climatologia e meteorologia, identificou-se preponderância da associação de "inundações" às mudanças climáticas, enquanto na geomorfologia e geociências em geral, a associação mais comum ocorre entre a variável hidrológica do escoamento superficial e a impermeabilização urbana. Dentre as produções nacionais, as pesquisas são mais comumente desenvolvidas utilizando técnicas de geoprocessamento e pesquisa histórica-documental, enquanto o uso de modelagem é dominante apenas na área da hidrologia. Os resultados corroboram com a hipótese original de trabalho, que considerou que as diferentes áreas do conhecimento diferem nas metodologias para abordagem do problema e, portanto, na identificação de variáveis causais, raramente considerando aquelas menos usuais em sua abordagem.

Palavras-chave: Risco; inundação; análise forense; mapeamento bibliométrico.

Abstract: In a broader research context, focused on the investigation of causal factors for the occurrence of floods in urbanized basins in São Paulo, a meta-analysis research was carried out on studies that focused on urban floods in different perspectives of analysis of related scientific fields (geomorphology, climatology, hydrology, etc.). It was sought to identify relationships between more associated concepts, more used methodologies and more pointed causal factors. The meta-analysis was carried out in two stages: a more extensive one, based on the worldwide base of Web of Science journals, and a more detailed one, on national bases of journals and repositories of thesis and dissertations. The data from the first stage were analyzed using bibliometric mapping and those from the second were indexed and analyzed qualitatively. The representativeness of researches on urban flooding originating in countries of humid tropical environment was considered significantly low. In the sub-area of climatology and meteorology, there is a preponderance on the association of "floods" with climate change, while in geomorphology and geosciences in general, the most common association is set between the hydrological variable of runoff and urban impermeabilization. Among national studies, research is most commonly carried out using geoprocessing techniques and historical-documentary research, while the use of modeling is dominant only in the area of hydrology. The results corroborate the original working hypothesis, which considered that the different areas of knowledge differ in methodologies for approaching the problem and, therefore, in the identification of causal variables, rarely considering those less usual in their approach. 
Keywords: Risk; floods, forensic analysis; bibliometric mapping.

\section{Introdução}

O processo de urbanização da cidade de São Paulo foi historicamente marcado por transgressões legais, planejamento territorial ineficiente e apropriação de compartimentos geomorfológicos cujas características originais, principalmente as de planaltos dissecados e amplas planícies meândricas do meio tropical úmido, criaram específicos e permanentes cenários de risco, dentre esses os de enchentes e inundações.

Considerando o caráter permanente e complexo desses riscos ao longo da história recente da cidade de São Paulo, considera-se necessário avaliar a natureza dos estudos desenvolvidos na temática, em especial daqueles que envolvem análises integradas ou diferentes variáveis do meio físico na busca de identificação de variáveis causais e ação dos agentes envolvidos. Esta busca por causas diversas, agentes envolvidos e seu peso ponderado na efetivação de um risco reconhecido é atualmente denominada de "análise forense" de inundações urbanas. De acordo com a proposta da metodologia forense (OLIVER-SMITH et al, 2016), o primeiro passo e objetivo dessa metodologia é o de investigar fatores identificados como causas de episódios de inundações em estudos realizados em diferentes áreas que abordam o tema como, por exemplo, mas não unicamente, a geomorfologia e a climatologia.

A geomorfologia possui abordagens e referenciais adequados a uma visão espacial integrada e ao mesmo tempo analítica no exame de mudanças em sistemas físicos de ambientes urbanos, incluindo a perspectiva histórica dessas mudanças associadas às ações antrópicas (RODRIGUES, 1997, 1999, 2004, 2010, 2015, LUZ e RODRIGUES, 2015, GURNELL et al, 2003, CRUTZEN, 2002). A temática das inundações interessa à geomorfologia não só como evento formativo naturalmente integrante de sistemas hidrogeomorfológicos, mas também como evento constituidor de riscos cuja intensidade e recorrência são intensificados pela urbanização.

Associadamente, o fenômeno de inundação também representa a repercussão de um fenômeno atmosférico que, na geomorfologia, pode ser subutilizado em função da indisponibilidade de dados em intervalos coerentes com as escalas espaciais e temporais de interesse. A compreensão da gênese e repercussão dos fenômenos atmosféricos de interesse à temática das inundações, concentra-se na análise das chuvas intensas ou concentradas sobre superfícies de sistemas morfológicos intensamente modificados, sendo um dos focos da perspectiva analítica do clima urbano (MONTEIRO, 1976). Quando este é considerado um produto da interação entre a atmosfera e um espaço urbano transformado e apropriado diferencialmente, passa a ser dotado de uma dimensão socioambiental (SANT'ANNA NETO, 2011). Isto, pois, é observável que, enquanto o clima obedece a controles superiores (escalas global e zonal), a ação indireta do antrópico se realiza com a concentração de suas atividades onde seus agentes aglomeram-se demograficamente e produzem economicamente (MONTEIRO, 2009).

Considerando as particularidades desses dois campos do conhecimento, é importante reconhecer que diferentes disciplinas respondem a diferentes estruturas de recompensa. Isso é, objetivam diferentes produtos de pesquisa. A progressão das especializações se expandiu recentemente para incluir ao estudo de desastres, especialidades com diversas dimensões sociais, econômicas, culturais e psicológicas (OLIVER-SMITH et al, 2016). O círculo dos conhecimentos e práticas relevantes se estende ainda mais para campos de expertise profissional como as engenharias e a medicina. Se considerarmos também as investigações com fins periciais, outros campos de expertise, como direito, políticas públicas e comunicações são necessariamente envolvidas. Portanto, é evidente a necessidade de metodologia integradora para um estudo de temática tão transversal que, independentemente do canal de interpretação utilizado, sempre tem origem em sistemas físicos e que requerem, portanto, partida em abordagens comumente utilizadas e válidas para esses campos.

$\mathrm{Na}$ metodologia forense, a forma de responder "quais são" e "como" geralmente são identificadas as causas de um tipo específico de risco, se dá através da metanálise. A metanálise é a revisão sistemática da literatura utilizada para identificar e avaliar descobertas relevantes através de diferentes estudos (BURTON, 2010). Trata-se, por exemplo, de estudo multivariado e estatístico de centenas de pesquisas sobre inundações ocorridas em contexto urbano semelhante em busca dos fatores causais. A metanálise é comumente utilizada como procedimento para sistematizar resultados de estudos similares baseado em um modelo de pesquisa consistente.

Por esse meio, o objetivo deste estudo é investigar os fatores que foram identificados como causas de episódios de inundações urbanas em estudos realizados em diferentes áreas que abordam o tema, através da revisão e mapeamento bibliométrico. Temos a hipótese de que estudos de áreas específicas tendem a 
identificar como fatores causais exclusivamente fenômenos estudados naquela área especializada, tendendo a não contemplar fatores críticos abordados por outras áreas.

Com esta abordagem analítica há potencial para investigação sistemática de desastres onde as descobertas dos estudos de caso ou observações de pesquisa são suficientemente comparáveis para uma ou múltiplas variáveis. Como apontam Oliver-Smith et al (2016), a maioria dos estudos adota a perspectiva meta analítica para atingir e avaliar padrões consistentes de causas, fatores condicionantes e resultantes da ocorrência de eventos naturais e desastres através de uma grande quantidade de categorias definidoras. Isso é particularmente relevante para um tipo de risco interdisciplinar entre ciências da Terra, em que cada uma tenderá a dar maior relevância a variáveis de sua expertise ao longo dos estudos, enviesando potencialmente a identificação de fatores causais e suas relevâncias em diversos contextos do meio físico.

\section{Materiais e métodos}

Para execução da metanálise sobre riscos e episódios de inundações em condições análogas à área de estudo desta pesquisa (a cidade de São Paulo), primeiramente foram levantadas produções científicas a respeito de inundações urbanas na base Web of Science. Considerando a hipótese que se busca testar com a aplicação deste método, a de que estudos enquadrados na área da climatologia tendem a identificar como fatores causais variáveis meteorológicas e estudos da geomorfologia e geociências tendem a apontar como causas as mudanças no uso do solo e ocupação de áreas suscetíveis, a busca na base de dados foi realizada com diferentes combinações dos termos: floods, flash floods, urban floods, civil engineering, geomorphology, meteorology, climatology, geociences, hydrology. A escolha por esses termos buscou englobar as duas adjetivações de inundações que mais interessam (urban e flash) e as cinco áreas com maior número de produções para o termo "floods".

Considerando a quantidade de resultados encontrados para a palavra-chave "Floods", cerca de 20.000, considerou-se que os filtros de palavras-chave e de áreas de concentração das publicações deveriam ser de tal forma combinados a fim de obterem correspondência em ao menos $10 \%$ desse total, percentual referente à proporção de estudos que tratam de inundações urbanas. Esta parcela do universo de publicações pode ser considerada representativa com base no alto grau de transdisciplinaridade e variação de enfoques esperados entre estudos desta temática (OLIVER-SMITH, 2016). Com o objetivo de categorização, as combinações na Tabela 1 apresentaram os resultados mais representativos para a discriminação das publicações sobre a temática entre as diferentes áreas de concentração.

Tabela 1: Concatenação de critérios utilizados para levantamento bibliométrico.

\begin{tabular}{cccc}
\hline Palavra-chave 1 & Palavra-chave 2 & Filtro de área & Resultados \\
\hline Flash floods & Urban & Nenhum & 414 \\
Floods & Urban & Engenharia Civil & 231 \\
Floods & Urban & Geociências & 1424 \\
Floods & Urban & Meteorologia & 704 \\
Floods & Urban & Geomorfologia & 241 \\
TOTAL & & & 2106 \\
\hline
\end{tabular}

Nenhuma outra combinação de palavras-chave correlatas ao tema obteve mais de 200 resultados em cinco categorias de áreas diferentes, permitindo a concatenação de critérios e filtros utilizados A substituição do termo "flood" por outros termos como "overflow" ou "hydrologic extreme" obteve estudos de contextos muito diferenciados e menos relacionados ao termo "urban" (menos de 10\% do total).

Os cinco conjuntos de publicações resultantes das buscas foram exportados com resumos completos de informações (títulos, lista de autores, lista de palavras-chave, resumo, instituição de pesquisa, agência financiadora, ano de publicação, etc.). Com vistas à maximização do tempo de análise do volume bibliográfico levantado, os dados indexados foram interpretados através de mapeamentos bibliométricos produzidos pelo software VOSviewer. Foram empregados os parâmetros descritos por Melo \& Queiroz (2016): a distância entre os elementos, inversamente proporcional à conexão indicando maior proximidade entre dois ou mais elementos; e o tamanho dos símbolos, indicando a relevância (quantitativa) para o universo amostrado.

Dada a baixa quantidade de estudos nacionais obtidos neste levantamento (apenas um), o que já era esperado, uma segunda etapa de metanálise foi executada com procedimentos diferentes. Considera-se a que a maioria das publicações sobre riscos e episódios de inundação no Brasil encontram-se em língua portuguesa, estando, portanto, em uma "zona de sombra" para ferramentas de busca em bases de dados como 
as empregadas na primeira etapa. A busca por estas publicações foi então realizada a partir de uma seleção de bancos de teses/dissertações e periódicos abertos considerados promissores, pela quantidade de estudos publicados sobre a temática nos últimos 10 anos, sendo fichados estudos apresentando: sua área do conhecimento, fatores causais identificados, principais métodos e conceitos utilizados. Tal seleção se deu com etapa preliminar de sondagem de acordo com a disponibilidade de consulta digital a acervos, sendo preferidas, assim, os seguintes tipos de fontes: 1- Bibliotecas de instituições nacionais com ampla produção reconhecida na temática de riscos a inundações; 2 - Periódicos e anais de eventos especializados na temática de riscos a inundações e eventos extremos fluviais e de precipitação. Ao final desta segunda etapa, foi feito fichamento de 180 estudos, podendo ser considerado conjunto representativo para o tipo altamente específico de análise sobre fatores causais.

Especificamente, foram consultados:

Tabela 2: Repositórios e periódicos consultados para fichamento de resumos.

\begin{tabular}{ll}
\hline \multicolumn{1}{c}{ Repositórios } & \multicolumn{1}{c}{ Periódicos } \\
\hline Teses USP & Revista Brasileira de Geomorfologia \\
Produção Científica e Intelectual Unicamp & Revista Brasileira de Recursos Hídricos \\
Institucional da UNESP & Revista do Departamento de Geografia da USP \\
Biblioteca Digital de Teses UFPR & Revista Geo-USP: Espaço e Tempo \\
Institucional UFScar & Revista Brasileira de Climatologia \\
Institucional UFMG & Anuário IGEO-UFRJ \\
Institucional UFSC & Journal of Hydrology \\
Institucional UFG & REGA - Revista de Gestão de Água da América Latina \\
Institucional UFES & International Journal of Climatology \\
Institucional UFRGS & Natural Hazards \\
Publicações IPT/SP & Geomorphology \\
& Revista Geografia de Rio Claro \\
& Quaternary and Environmental Geosciences \\
& Revista GEO/UERJ \\
& Climep: Climatologia e Estudos da Paisagem \\
& Hydrological Processes \\
\hline
\end{tabular}

Nestes conjuntos de publicações e revistas foram utilizadas como palavras-chave de busca: inundações, enchentes, drenagem urbana, eventos hidrológicos extremos, águas pluviais urbanas e, complementarmente, urban floods, flash floods, stormwater management, hydrological extremes.

Dos resultados, foram coletados, analisados e fichados somente os estudos realizados sobre impactos e riscos de inundações, excluindo-se aqueles ocasionados por marés, em bacias urbanas de cidades do meio tropical úmido. Ao final do levantamento, foi realizado fichamento de 180 estudos que atenderam aos critérios de busca, podendo ser considerado conjunto representativo para o tipo altamente específico de análise e por constituir quantidade comparável aos resultados da concatenação de filtros da primeira etapa da pesquisa.

\section{Resultados e discussões}

\section{1 base web of science}

Com base no levantamento mais extensivo, realizado na base de dados Web of Science, destaca-se a concentração de estudos sobre inundações urbanas realizadas na área da meteorologia e climatologia, representando um terço do total. Dentre estes, as palavras-chave "mudanças climáticas" e "vulnerabilidade" se evidenciam, sendo utilizadas em $15 \%$ dos estudos analisados. Uma vez que não tenha sido realizada leitura minuciosa de toda o volume de publicações encontradas, não foi possível afirmar correspondência conceitual e escalar no uso da expressão "mudanças climáticas" entre estes. No entanto, é preciso considerar que foram selecionados apenas estudos sobre meio urbano, sendo questionável se todos eles utilizaram o conceito de acordo com as escalas geográficas do clima, em que mudanças climáticas são constatáveis com análises em escalas espaciais zonais e temporais milenares. Os resultados da busca demonstram a predominância de estudos com tema alinhado às agendas internacionais e globais de pesquisa e políticas públicas (Figura 1), em que a identificação de efeitos de mudanças climáticas sobre tendências e frequência de eventos de precipitação extremos é tema prioritário para financiamento (ALCANTARA-AYALA, 2010). 


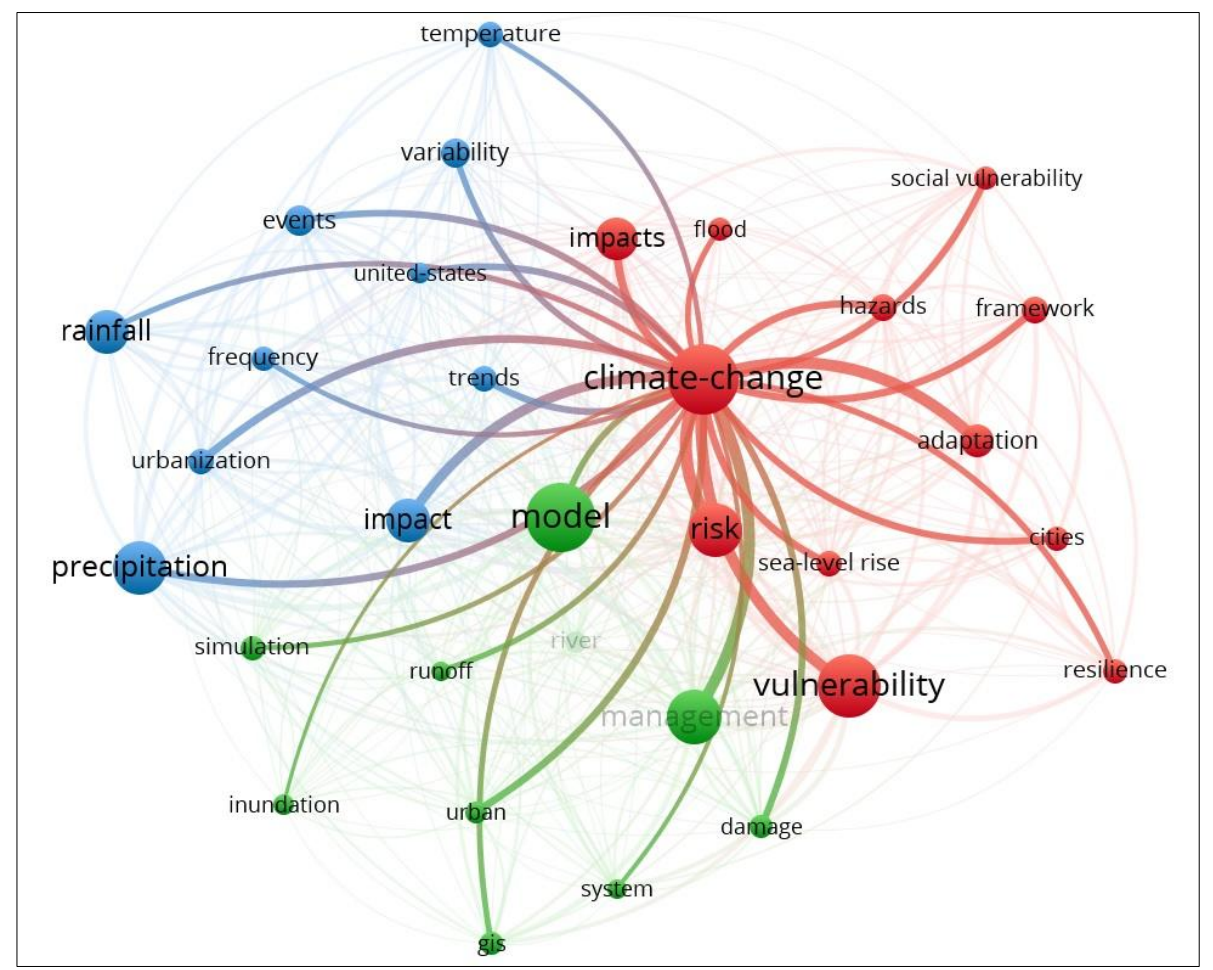

Figura 1: Mapeamento bibliométrico das palavras-chaves encontradas em publicações sobre inundações urbanas na área Meteorologia/Climatologia.

Quanto às publicações levantadas utilizando o filtro de área da Geomorfologia, não foi identificada palavra-chave com correlação de destaque em parcela grande das publicações como no caso anterior. Os termos mais empregados de forma associada foram o de "urbanização" (em 9,5\% das publicações e "runoff" em $8 \%$ das publicações), o que nos permite inferir foco dado às modificações no balanço infiltração/escoamento superficial decorrentes das intervenções de uso e ocupação do solo pelo processo de urbanização (Figura 2). Assim, no caso dos estudos deste campo do conhecimento científico sobre inundações, a questão das mudanças climáticas aparece associada mais fortemente ao desenvolvimento do ambiente urbano e as inundações tenderam a ser consideradas como problemas associadas aos conceitos de bacias hidrográficas e seu gerenciamento.

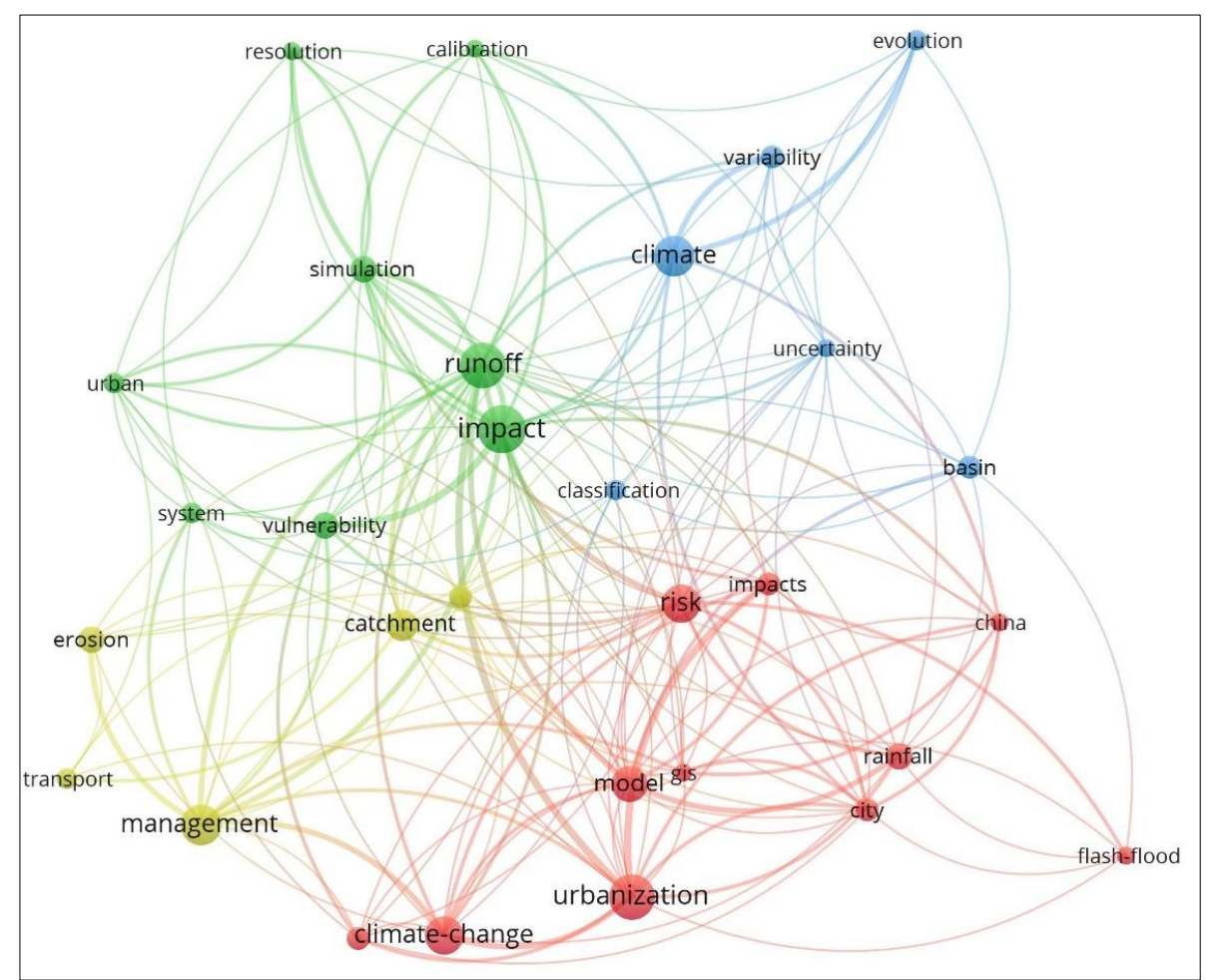

Figura 2: Mapeamento bibliométrico das palavras-chaves encontradas em publicações sobre inundações urbanas na área Geomorfologia. 
Isso torna interessante a observação de que quando se consideram as publicações encontradas sobre inundações urbanas na área de Engenharia Civil, os estudos tendem a se direcionar mais às modelagens de processos em redes e sistemas urbanos. Assim como nos resultados para a área de geomorfologia, as inundações nesse campo de aplicação são consideradas associadamente aos processos hidrológicos e hidráulicos de superfície (Figura 3). Destaca-se também que a palavra-chave "mudanças climáticas" se faz presente, mas a variável "precipitação" possui baixa correspondência.

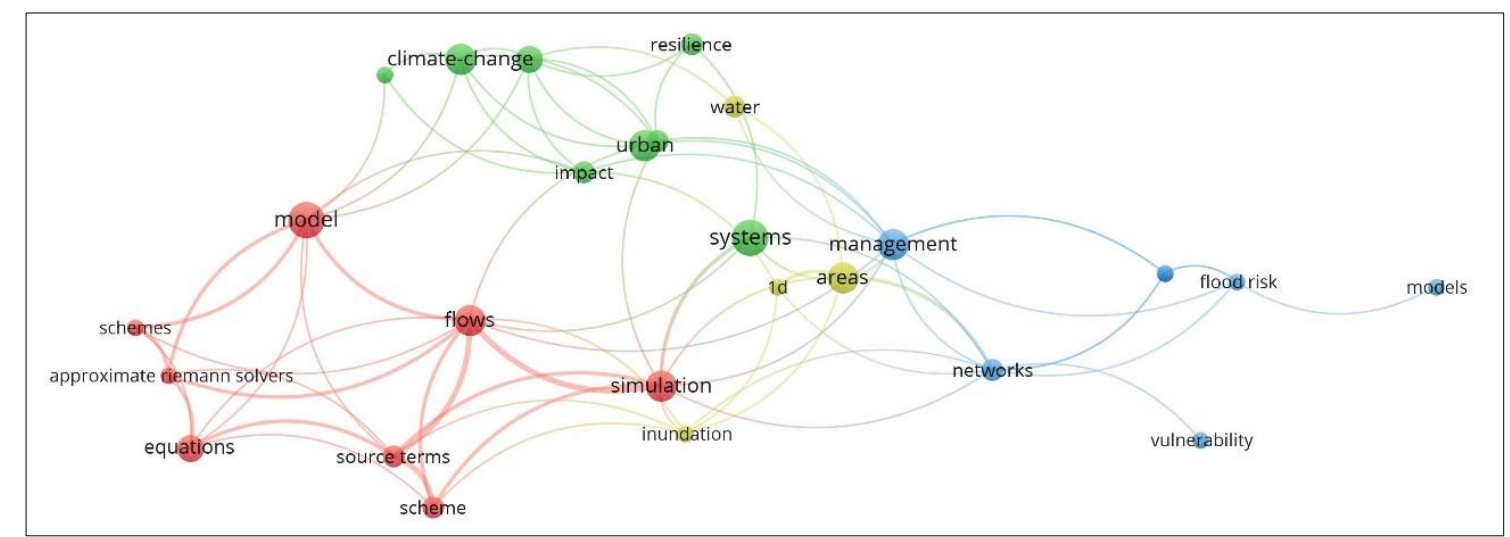

Figura 3: Palavras-chaves encontradas em publicações sobre inundações urbanas na área Engenharia Civil.

Em comum entre as três áreas de conhecimento filtradas, os termos "modelo", "calibração" e "simulação" permitem evidenciar a emergência de técnicas de modelagem em meio aos estudos sobre inundações independentemente da área de concentração. $O$ conceito de modelo vem mais frequentemente (aproximadamente 20\% das ocorrências) associado aos conceitos de "previsão" (forecasting) e "resolução", presumivelmente em decorrência da melhoria na maior disponibilidade de dados primários com resoluções espaciais e temporais cada vez maiores.

Com o mapeamento do uso das palavras-chave em estudos sobre o tipo específico de inundações "flash floods" classificados por ano de publicação, foi possível notar, a correlação de períodos com maior concentração de uso deste termo com o termo "modelo" associado a "precipitação" e "runoff" (Figura 4). Logo, destaca-se esta como a tipologia de fenômeno mais frequentemente investigada pelo uso de modelos hidrológicos associados à análise da precipitação.

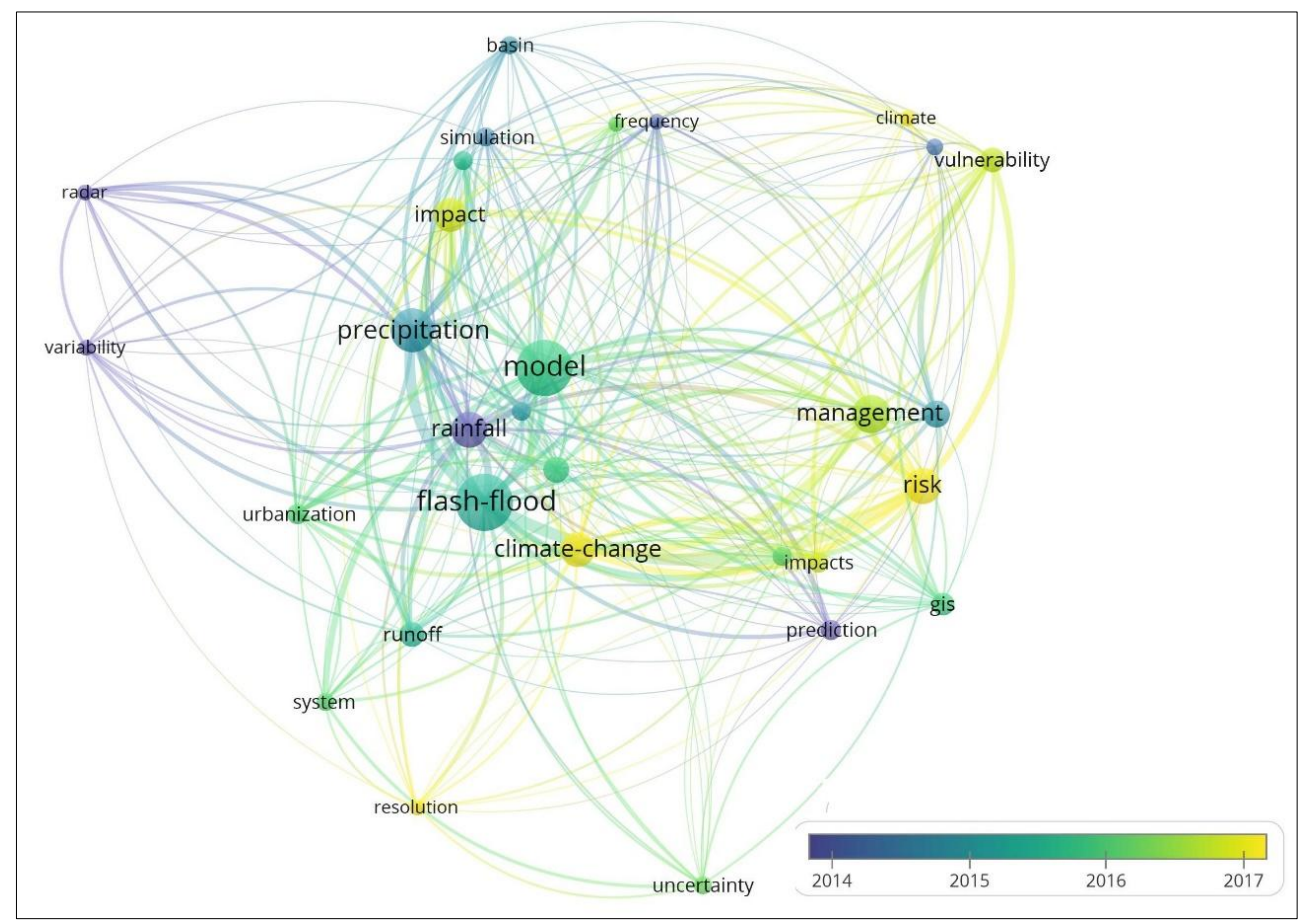

Figura 4: Mapeamento bibliométrico das palavras-chaves encontradas em publicações sobre flash floods, independente da área de concentração, por ano de publicação. 
Dentre os resultados do levantamento realizado sobre estudos que traziam especificamente a palavrachave flash floods, destacam-se: a concentração destes nos Estados Unidos (mais da metade das publicações) e a posição periférica ocupada pelos países do meio tropical úmido, como México e Colômbia, devido à baixa quantidade de estudos desenvolvidos com este termo específico (Figura 5).

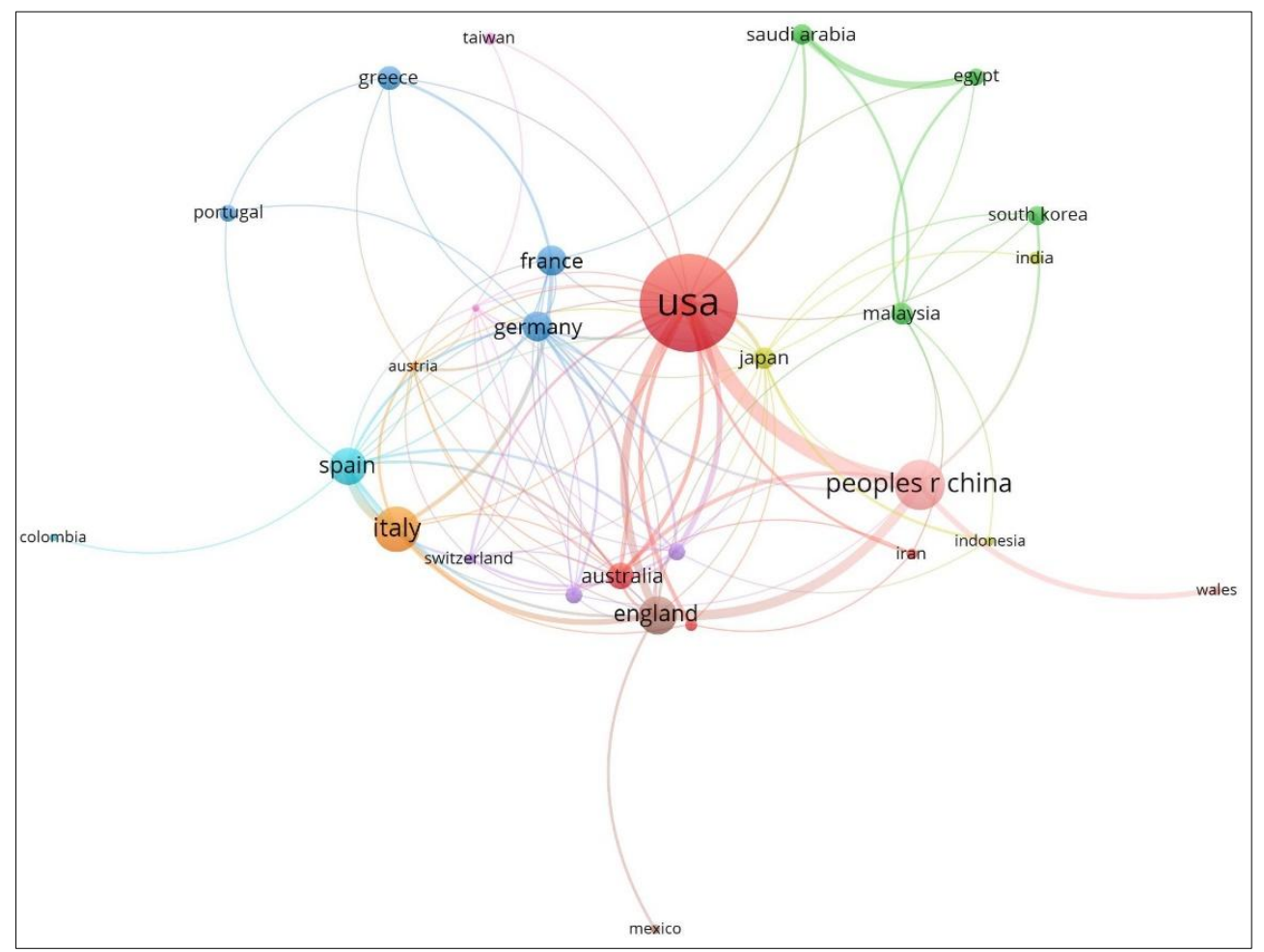

Figura 5: Mapeamento bibliométrico dos países de origem das publicações com o termo flash floods.

Com isso, foi possível concluir a incipiência de estudos com identificação de flash floods no contexto de cidades como São Paulo, bem como torna-se evidente a baixíssima representatividade de estudos realizados no Brasil sob o escopo (apenas 1 resultado). Para suprir esta deficiência, de maneira que a metanálise não ignorasse justamente os estudos realizados sobre áreas mais semelhantes à área de estudo, a segunda etapa de levantamentos e fichamentos constituiu universo de análise com fontes de produções científicas nacionais.

\subsection{Pesquisas nacionais}

Foram levantadas 171 produções científicas sobre inundações urbanas realizadas no Brasil, entre os anos 1983 e 2019, das quais 132 são dos últimos 10 anos. Este recorte se dá exclusivamente como resultado da disponibilidade de material nas fontes consultadas. Também foram consideradas publicações sobre estudos em Bangladesh (1), China (4), Índia (3) e Singapura (1), dada a similaridade das localidades analisadas com a presente área de estudo. Do total de 180 estudos, nota-se que $41 \%$ dos estudos não utilizaram o recorte de bacias hidrográficas. Isso permite notar o como ainda há grande quantidade de estudos sobre impactos de um sistema físico desenvolvidos a partir de recortes administrativos (município, bairro, etc.).

Com a classificação destas publicações em áreas do conhecimento em que melhor se inserem suas respectivas s técnicas e conceitos empregados, ficou evidente que os estudos estão concentrados em duas áreas (Figura 6): na área da hidrologia (total de 80 publicações) e da geomorfologia (27 publicações).

Justifica-se a separação da Geografia quanto à Climatologia e Geomorfologia pelo maior foco dado em alguns estudos a métodos e técnicas da geografia urbana e cartografia mais geral. Pela classificação realizada, destaca-se a baixa concentração de publicações sobre inundações urbanas enquadradas especificamente nas áreas de Climatologia e Geomorfologia, sendo preteridas por métodos e técnicas da Hidrologia. Nesta área prevalente, nota-se a menor concentração de estudos que apresentaram em conclusão a identificação de fatores causais das inundações (3/4 deles, Figura 7). Em sua maioria, os estudos desta área priorizaram a aplicação de modelos hidráulicos e hidrológicos para simulação de inundações potenciais ou reconstituição de eventos passados, havendo preocupação predominante sobre sistemas e não fatores. Desta forma, a impermeabilização e falhas em sistemas de drenagem construídos são os fatores causais mais citados entre os estudos de hidrologia sobre inundações urbanas. 


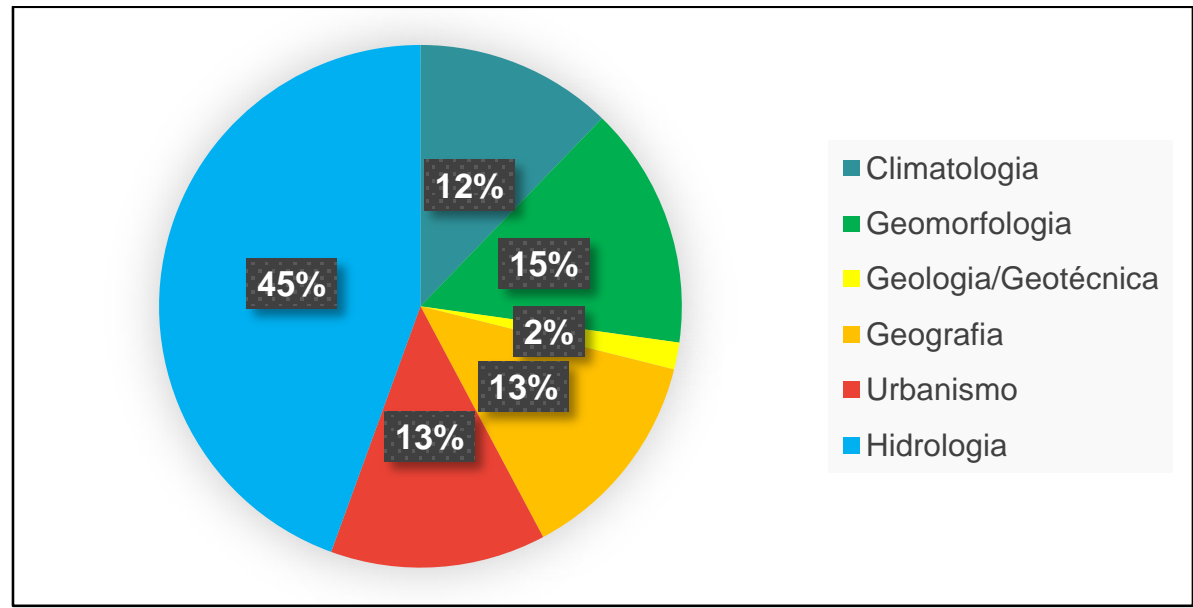

Figura 6: Concentração de estudos classificados por área.

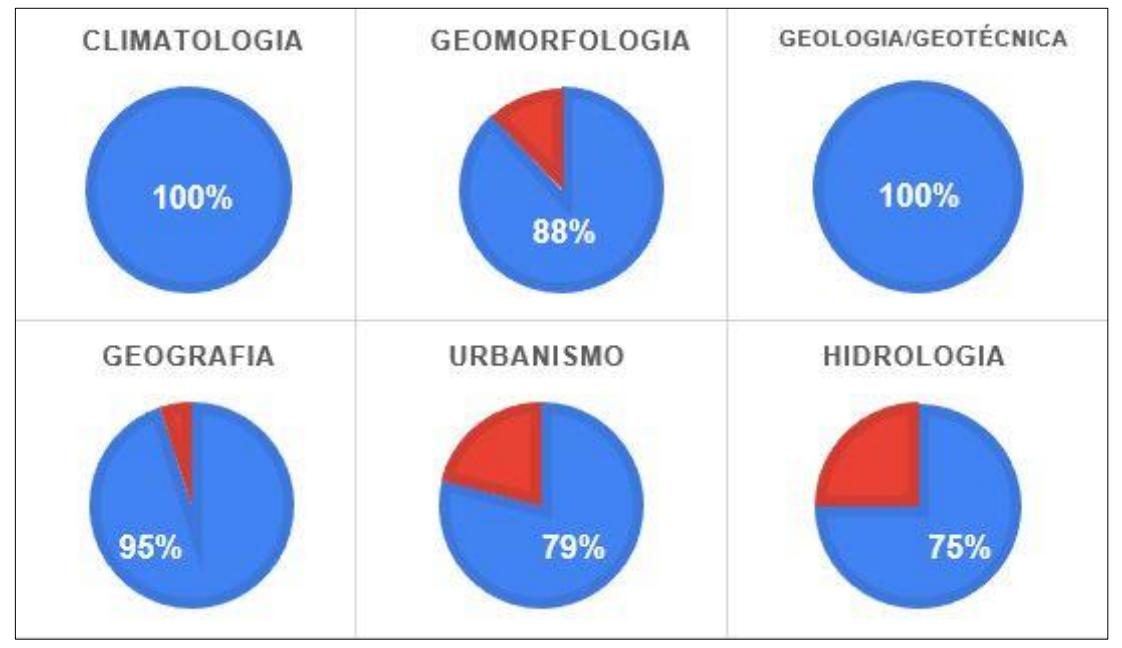

Figura 7: Porcentagem de estudos por área /fatores causais de inundações.

Todos os estudos analisados classificados na área de conhecimento da Climatologia identificaram fatores causais, sendo o mais citado "eventos de precipitação intensa". Isso ocorre devido notada preferência de abordagem a episódios particulares, bem como é o resultado esperado para estudos que tiveram, em quase todos os casos, o uso de análise climatológica como principal ferramenta analítica. Esse tipo de característica do universo de estudos em climatologia, torna ainda mais relevantes as tendências identificadas dos estudos em geomorfologia de não apresentar as mesmas técnicas e os mesmos tipos de fatores causais. Nesta área foi privilegiada a utilização de técnicas de geoprocessamento e, na maioria desses casos, as mudanças no uso do solo e em canais fluviais foram as causas mais relacionadas às inundações urbanas (Tabela 3).

Tabela 3: Relação de técnicas mais utilizadas e fatores causais mais citados de acordo com as áreas.

\begin{tabular}{llrlc}
\hline \multirow{2}{*}{ Área } & \multicolumn{4}{c}{ Técnica mais empregada } \\
\cline { 2 - 5 } & Mais citada/utilizada & $\mathbf{n}^{\mathbf{0}}$ & Segunda mais citada/utilizada & $\mathbf{n}^{\mathbf{0}}$ \\
\hline Hidrologia & Modelagem Hidráulica & 59 & Geoprocessamento & 34 \\
Climatologia & Análise climatológica & 19 & Pesquisa Histórica-Documental & 8 \\
Geomorfologia & Geoprocessamento & 14 & Pesquisa Histórica-Documental & 12 \\
Geologia/Geotécnica & Geoprocessamento & 3 & Pesquisa Histórica-Documental & 1 \\
Geografia & Geoprocessamento & 14 & Pesquisa Histórica-Documental & 13 \\
Urbanismo & Pesquisa Histórica-Documental & 19 & Geoprocessamento & 5 \\
\hline & & Fator causal identificado & 14 \\
\hline Hidrologia & Impermeabilização do solo & 22 & Falha na drenagem urbana & 12 \\
Climatologia & Eventos de precipitação intensa & 18 & Ocupação de áreas suscetíveis & 8 \\
Geomorfologia & Mudanças no uso do Solo & 19 & Mudanças em canais fluviais & 1 \\
Geologia/Geotécnica & Ocupação de áreas suscetíveis & 2 & Impermeabilização do solo & 8 \\
Geografia & Mudanças no uso do Solo & 17 & Ocupação de áreas suscetíveis & 10 \\
Urbanismo & Mudanças no uso do Solo & 11 & Ocupação de áreas suscetíveis & \\
\hline
\end{tabular}


Uma coincidência entre todas as áreas é a representatividade de estudos que utilizaram técnicas de pesquisa histórica e documental (potencialidade já identificada por RODRIGUES, 1997, 2004, 2010, 2015, LUZ \& RODRIGUES, 2015, GURNELL et al, 2003). Sua recorrência aponta para a importância dada nos estudos à investigação de fenômenos e impactos de ocorrência constatada, sendo um aspecto relativamente preterido no caso dos estudos relativos à Hidrologia. Isso se atribui à natureza mais estimativa dos estudos levantados, em sua maioria pautados em análises de tempos de recorrência.

\section{Conclusões}

Apesar de ser identificada uma menor concentração de estudos nas áreas de Climatologia e a Geomorfologia do que o esperado inicialmente, foi possível notar a diferença existente entre as técnicas empregadas e consequentemente nos fatores causais apontados. Identificou-se preferência, nos estudos de cada área, pela investigação de variáveis relacionadas aos seus objetos (precipitação no caso da climatologia e morfologias no caso da geomorfologia). Assim, os fatores causais que estes identificaram não são exclusivos, mas tendem a ser analisados desassociados, mesmo quando se pretende uma abordagem sistêmica. Isso reforça a importância de emprego das ferramentas que, através desta metanálise, foram identificadas como comuns às diferentes áreas (como o geoprocessamento), pela capacidade de melhor integrarem diferentes variáveis do sistema físico.

Destacou-se também a concentração de estudos sobre inundações urbanas realizadas na área da meteorologia e climatologia, representando um terço do total. Dentre estes, a palavra-chave "mudanças climáticas" apresenta-se como a mais utilizada, enquanto "eventos de precipitação intensa" aparece como o fator causal mais apontado (tendência também identificada por ALCATARA-AYALA, 2010). Já na área da Geomorfologia, os termos mais associados são "urbanização" e "runoff" apontando mais frequentemente "mudança no uso do solo", de forma genérica, como fator causal e com maior emprego de técnicas de geoprocessamento. É notável que $41 \%$ dos estudos fichados não focavam sua análise sobre bacias hidrográficas e, a modelagem hidráulica, aparece como a técnica mais empregada nas áreas da hidrologia e engenharia civil.

$\mathrm{O}$ estudo demonstrou lacunas quanto ao desenvolvimento de pesquisas interdisciplinares voltadas à compreensão de ocorrências de inundações/enchentes em áreas urbanizadas de forma geral e, particularmente, para o meio tropical úmido, apontando para a necessidade de se trabalhar em algumas dessas interlocuções disciplinares. As razões para essas lacunas serem problemáticas são diversas e ficam mais evidentes em estudos forenses. Uma dessas razões é o fato de que, em paisagens altamente modificadas, não apenas os fatores desencadeadores comumente tratados em estudos específicos sejam cambiantes em termos de sua importância relativa, mas também são cambiantes seus valores limiares para o desencadeamento de inundações, que apresentam valores diversos daqueles reconhecidos para sistemas naturais equivalentes. Este é o caso demonstrado em estudos como os de Rodrigues et al (2014), Moroz Caccia-Gouveia (2010), Simas et al (2014), Simas \& Rodrigues (2016) e Hooke (1999, 2006, 2015). Em todos eles, as variáveis relativas à morfologia antrópica detalhada assumiram uma maior ou equivalente relevância na ocorrência e localização do processo em questão, quando comparadas às variáveis climatológicas. Essas últimas, contudo, ainda apresentam valores limiares nos quais o processo de inundação ocorrerá, independentemente de cobertura vegetal e uso da terra urbano detalhado. Outra razão relevante para o fato dessas lacunas serem problemáticas é o fato de que nos estudos avaliados, os fatores causais identificados tendem a deixar de fora a gestão do meio físico, os processos históricos e agentes socioeconômicos. Tais constatações são condizentes com os resultados de Mantovani \& Rodrigues (2018), que apontam como a subutilização de arcabouços metodológicos e conceitos próprios de uma área do conhecimento podem conduzir a resultados inadequados do ponto de vista forense.

Em conclusão, a metanálise revelou a carência de estudos interdisciplinares sobre inundações em cidades do meio tropical úmido, o que pode servir de explicação para a formulação de medidas tanto estruturais quanto não estruturais que, historicamente, são implementadas tendo efeitos limitados.

\section{Referências}

ALCANTARA-AYALA. I. Geomorphology and disaster prevention. In: ALCANTARA-AYALA, I. GOUDIE, A. S. Geomorphological Hazard and Disaster prevention. New York: Cambridge University Press, 2010. p. 269-278. 
BURTON, I. Forensic Disaster Investigations in Depth: A New Case Study Model. Environment: Science and Policy for Sustainable Development, v. 52, n. 5, p. 36-41, 2010.

CRUTZEN, P. J. Geology of mankind. Nature, 415: doi 101038/415023a

FUNDAÇÃO CENTRO DE TECNOLOGIA HIDRAULICA - FCTH. Diretrizes Básicas para projetos de drenagem urbana no município de São Paulo. São Paulo, 1999. Disponível em:http://www.fcth.br/public/cursos/canaismares/md.pdf. Acesso em: 10/09/2015.

GURNELL, A. M., PERIY, J. L, PETTS, G. E. Using Historical Data in Fluvial Geomorphology. In.: Tools in fluvial geomorphology, Org.: Kondolf, G. M., Piegay, H. New Jersey: John Wiley \& sons, 2003.

HOOKE, J. M. Decades of change: contributions of geomorphology to fluvial and coastal engineering and management. Geomorphology, 31(1-4), 1999. p. 373-389.

HOOKE, J. M. Hydromorphological adjustment in meandering river systems and the role of flood events. IAHS-AISH Publ, 2006. p. 127-135.

HOOKE, J. M. Variations in flood magnitude-effect relations and the implications for flood risk assessment and river management. Geomorphology, v. 251, 2015. p. 91-107.

LUZ, R. A., RODRIGUES, C. Anthropogenic changes in urbanised hydromorphological systems in a humid tropical enrinment: River Pinheiros, Sao Paulo, Brazil. Stuttgat: Zeitschrift für Geomorphologie, Vol. 59, Suppl. 2, p. 109-135, 2015.

MANTOVANI, J. C., RODRIGUES, C. Geomorfologia aplicada à análise forense: uma avaliação necessária. São Paulo: Revista do Departamento de Geografia. Volume Especial do II Workshop do Programa de Pós-Graduação em Geografia Física, 2018.

MELO, A. V. F., QUEIROZ, A. P. Bibliometric mapping of papers on geographical information systems (2007-2016). Curitiba: Boletim de Ciências Geodésicas, vol. 23, n. 3. 2019.

MONTEIRO, C. A de F. A dinâmica climática e as chuvas no estado de São Paulo: Estudo geográfico sob a forma de Atlas. São Paulo: USP/Igeog, 1973.

MONTEIRO, C. A de F. Teoria e Clima Urbano. São Paulo: Universidade de São Paulo, Instituto de Geografia, 1976.

MONTEIRO, C. A de F. Clima e excepcionalismo: Conjecturas sobre o desempenho da atmosfera como fenômeno geográfico. Editora da UFSC: Florianópolis, 1991.

MONTEIRO, C. A de F. Teoria e Clima Urbano. In.: Clima Urbano. Org: Monteiro, C. A. de F.; Mendonça, F. $1^{\text {a }}$ edição. São Paulo: Contexto, 2009. p. 9-69.

MOROZ - CACCIA GOUVEIA, I. C. Da originalidade do sitio urbano de São Paulo às formas antrópicas: aplicação da abordagem da Geografia Antropogênica na Bacia Hidrográfica do Rio Tamanduateí, na Região Metropolitana de São Paulo. Tese de doutorado em Geografia Física. Faculdade de Filosofia, Letras e Ciências Humanas, Universidade de São Paulo, 2010.

OLIVER-SMITH, A. Disaster Risk Reduction and Climate Change Adaptation: The View from Applied Anthropology. Human Organization, Vol. 72, No. 4, p. 275-282, 2013.

OLIVER-SMITH, A. ALCÁNTARA-AYALA, I., BURTON, I., LAVELL, A. M. Forensic Investigations of Disasters (FORIN): a conceptual framework and guide to research (IRDR FORIN Publication $n^{\circ} 2$ ). Beijing: Integrated Research on Disaster Risk, 2016.

RODRIGUES, C. Geomorfologia aplicada: Avaliação de experiências e de instrumentos de planejamento físico-territorial e ambiental brasileiros. Tese de doutorado (Geografia Física). Faculdade de Filosofia, Letras e Ciências Humanas, Universidade de São Paulo, 1997. 
RODRIGUES, C. On antropogeomorphology. In : Anais da regional Conference on geomorphology : Rio de Janeiro, 1999. p. 100-110.

RODRIGUES, C. A urbanização da metrópole sob a perspectiva da geomorfologia: Tributo às leituras geográficas. In. : Carlos, A. F. A. ; Oliveira, A. U. de (Org.) Geografias de São Paulo : Representação e crise da metrópole. Obra em 2v. São Paulo : Contexto, 2004. p. 89-115.

RODRIGUES, C. Avaliação do impacto humano da urbanização em sistemas hidrogeomorfológicos. Desenvolvimento e aplicação de metodologia na grande São Paulo. São Paulo: FFCHL-USP, Revista do Departamento de Geografia, n²0, p.111-125, 2010.

RODRIGUES, C. Atributos ambientais no ordenamento territorial urbano: o exemplo das planícies fluviais na metrópole de São Paulo. São Paulo: GEOUSP (Online), v. 19, n. 2, p.324-347, 2015.

RODRIGUES, C., MOROZ-CACCIA GOUVEIA, I. C., BERGES, B., LUZ, R. A. Geomorfologia urbana histórica e a avaliação da influência de fatores naturais e antrópicos na geração de eventos fluviais extremos em São Paulo - Brasil. In: Envibras 2014 Environnement et géomatique: approches comparées France Brésil, Rennes: Actes du Colloque, 2014.

SANT'ANNA NETO, J. L. Por uma Geografia do Clima: antecedentes históricos, paradigmas contemporâneos e uma nova razão para um novo conhecimento. São Paulo: Terra Livre, nº 17, p.49-62, 2001.

SANT'ANNA NETO, J. L. O clima urbano como construção social: da vulnerabilidade polissêmica das cidades enfermas ao sofisma utópico das cidades saudáveis. Revista brasileira de climatologia, ano 7, vol. 8, p.45-59, 2011.

SIMAS, I. T. H., RODRIGUES, C. Flood hazards analysis based on changes of hydrodynamic processes in fluvial systems of São Paulo, Brazil. Geophysical Research Abstracts. v. 18 Göttingen: Copernicus GmbH, 2016.

SIMAS, I. T. H., SANT'ANNA NETO, J. L., DUBREUIL, V. Application de la technique d'analyse rythmique à la vulnérabilité aux événéments pluviométriques extrêmes dans des quartiers urbains à São Paulo, Brésil. Actes du Colloque de l'Association Internationale de Climatologie, Dijón, 2014. p. 590598.

VAN ECK, N.J., WALTMAN, L. VOS: A New Method for Visualizing Similarities Between Objects. In: Decker, R., Lenz, H.J. (Org) Advances in Data Analysis. Studies in Classification, Data Analysis, and Knowledge Organization. Berlin, Heidelberg, 2007.

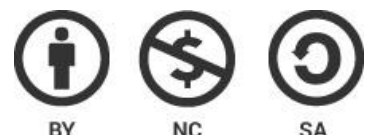

NãoComercial-CompartilhaIgual (CC BY-NC-SA). 